\title{
Dual trigger in normally-responding assisted reproductive technology patients increases the number of top-quality embryos
}

\author{
Yavuz Emre Şükür ${ }^{1}$, Hasan Ulubaşoğlu ${ }^{2}$, Fatma Ceylan İlhan ${ }^{3}$, Bülent Berker ${ }^{1}$, Murat Sönmezer ${ }^{1}$, Cem Somer Atabekoğlu' \\ Ruşen Aytaç ${ }^{1}$, Batuhan Özmen ${ }^{1}$
}

'Department of Obstetrics and Gynecology, Ankara University School of Medicine, Ankara; ${ }^{2}$ Department of Obstetrics and Gynecology, Ankara City Hospital, Ankara; ${ }^{3}$ Department of Obstetrics and Gynecology, Yenimahalle State Hospital, Ankara, Turkey

Objective: The feasibility of a gonadotropin-releasing hormone agonist (GnRHa) trigger in normal responders is still a matter of debate. The aim of this study was to compare the number of mature oocytes, the number of good-quality embryos, and the live birth rate in normal responders triggered by GnRHa alone, GnRHa and human chorionic gonadotropin (hCG; a dual trigger), and hCG alone.

Methods: A retrospective cohort study was conducted at the infertility clinic of a university hospital. Data from 200 normal responders who underwent controlled ovarian hyperstimulation and intracytoplasmic sperm injection with a GnRH antagonist protocol between January 2016 and January 2017 were reviewed. The first study group consisted of patients with cycles triggered by GnRHa alone. The second study group consisted of patients with cycles triggered by both GnRHa and low-dose hCG (a dual trigger). The control group consisted of patients with cycles triggered by $\mathrm{hCG}$ alone.

Results: The groups were comparable in terms of demographics and cycle characteristics. The numbers of total oocytes retrieved and metaphase II oocytes were similar between the groups. The total numbers of top-quality embryos were $3.2 \pm 2.9$ in the GnRHa group, $4.4 \pm 3.2$ in the dual-trigger group, and $2.9 \pm 2.1$ in the $\mathrm{hCG}$ group $(p=0.014)$. The live birth rates were $21.4 \%, 30.5 \%$, and $28.2 \%$ in those groups, respectively $(p=0.126)$.

Conclusion: In normal responders, a dual-trigger approach appears superior to an hCG trigger alone with regard to the number of top-quality embryos produced. However, no clinical benefit was apparent in terms of live birth rates.

Keywords: Assisted reproductive technology; Dual trigger; Final oocyte maturation; GnRH agonist; Normal response

Received: April 28, 2020 · Revised: July 9, 2020 • Accepted: July 14, 2020 Corresponding author: Yavuz Emre Şükür

Department of Obstetrics and Gynecology, Cebeci Hospital, Ankara University School of Medicine, Dikimevi, 06100 Ankara, Turkey

Tel: +90-312-595-87-01 Fax:+90-312-320-35-53 E-mail:yesukur@gmail.com

*This study was presented at the 25th World Congress on Controversies in Obstetrics, Gynecology \& Infertility (COGI) in Vienna, Austria, held from November 30 to December 2, 2017.

This is an Open Access article distributed under the terms of the Creative Commons Attribution Non-Commercial License (http://creativecommons.org/licenses/by-nc/4.0/) which permits unrestricted non-commercial use, distribution, and reproduction in any medium, provided the original work is properly cited.

\section{Introduction}

Mature oocytes are necessary for oocyte retrieval and fertilization, and final oocyte maturation is the key step in assisted reproductive technology (ART) cycles. Human chorionic gonadotropin (hCG) has been used for final oocyte maturation for many years and has been demonstrated to promote an increased pregnancy rate. However, the administration of hCG for final oocyte maturation results in supraphysiologic steroid levels in the luteal phase due to its long half-life and is consequently associated with an increased risk of ovarian hyperstimulation syndrome (OHSS) [1]. In addition, it has been sug- 
gested that an hCG trigger may have negative impacts on endometrial receptivity and embryo quality [2].

At the end of the last century, gonadotropin-releasing hormone agonists (GnRHas) were introduced to promote final oocyte maturation in GnRH antagonist cycles [3,4]. GnRHa has been documented to induce endogenous luteinizing hormone $(\mathrm{LH})$ and follicle-stimulating hormone (FSH) surges similar to the natural mid-cycle $\mathrm{LH}$ surge with a shorter duration and smaller amplitude than are associated with the administration of exogenous hCG, which may help to reduce the risk of OHSS [3,5]. The use of a GnRHa trigger may have possible benefits, including the induction of both an FSH and an $\mathrm{LH}$ surge and the possibility of retrieving more metaphase II (MII) oocytes than is expected with an hCG trigger [2,3,5]. Previously, the major drawback of a GnRHa trigger was LH depletion and the withdrawal of LH support of the corpus luteum, reflected by a reduced live birth rate and an increased miscarriage rate $[2,5]$. However, this problem has been overcome with intensive luteal phase support (LPS) and luteal-phase hCG administration [6].

As a result of developments in LPS, the concept of a GnRHa trigger has been fully established during the last decade in hyper-responder patients [6,7]. However, insufficient evidence is available regarding the impact of a GnRHa or a dual trigger on normal responders. A recent observational study reported cycle outcomes following a GnRHa trigger with exclusive hCG support in a progesterone-free luteal phase, and the results were comparable to those of cycles triggered by hCG [8]. This study was rare in that it supported a GnRHa trigger as a valid alternative in normal responders. However, a GnRH trigger is not yet a standard of care, and its feasibility in normal responders needs to be clarified.

The aim of the present study was to investigate whether differences were present in the number of oocytes collected, the number of top-quality embryos, and the live birth rate in normal responders triggered by GnRHa alone, GnRHa and hCG (a dual trigger), and hCG alone.

\section{Methods}

In the present retrospective cohort study, data from normal responders who underwent intracytoplasmic sperm injection following a cycle downregulated by a GnRH antagonist at a university-based infertility clinic between January 2016 and January 2017 were reviewed. The study was approved by the Ethical Committee of Ankara University School of Medicine (No. 08-341-16). In total, 214 patients who underwent intracytoplasmic sperm injection cycles with an antagonist protocol involving a starting dose of $225 \mathrm{IU}$ per day during the study period were selected from the hospital database. The inclusion criteria were the female patient being 18-40 years of age, a baseline FSH level of 3-15 IU/L, a baseline LH level above $3 \mathrm{IU} / \mathrm{L}$, a normal response to ovarian stimulation, and (as mentioned above) a starting dose of gonadotropin stimulation of 225 IU/day. Cases of female factor infertility (tubal factor, pelvic adhesions, or mild endometriosis), mild male factor infertility, and unexplained infertility were included in the analyses. A normal response was defined as the aspiration of 6-14 oocytes and a maximum oestradiol level lower than $2,500 \mathrm{pg} / \mathrm{mL}$. The exclusion criteria were secondary infertility, a body mass index over $30 \mathrm{~kg} / \mathrm{m}^{2}$, poor or hyper-response to ovarian stimulation, severe male oligozoospermia or azoospermia, the presence of any untreated thyroid dysfunction or hyperprolactinaemia, the presence of uterine abnormality, and the avoidance of fresh embryo transfer (freeze-all). After application of the inclusion and exclusion criteria, 200 patients were found to be eligible for analysis. Eleven patients who underwent a freeze-all cycle and three patients with abnormal thyroid function tests were excluded. For all women, only the first fresh cycles were included in the analyses.

For eligible participants, all data on ovarian stimulation and clinical outcomes were extracted from the database, and patients were divided into three groups according to the trigger method. The first study group consisted of patients with cycles triggered by $\mathrm{GnRHa}$ alone. An injection of $0.2 \mathrm{mg}$ triptorelin acetate (Gonapeptyl $0.1 \mathrm{mg}$; Ferring, Istanbul, Turkey) or $1 \mathrm{mg}$ (20 units in a tuberculin syringe of 5 $\mathrm{mg} / \mathrm{mL}$ injectable solution) leuprolide acetate (Lucrin $5 \mathrm{mg}$; Abbott, Istanbul, Turkey) was administered for final oocyte maturation in the GnRHa group. The second study group consisted of patients with cycles triggered by both GnRHa and hCG (the dual-trigger group). Those patients received an additional 1,500-IU hCG injection for final oocyte maturation (Pregnyl 5,000 IU; MSD, Oss, the Netherlands). The control group consisted of patients with cycles triggered by 10,000 IU hCG alone (the hCG group). The method of final oocyte maturation was based on the primary physician's preference. Additionally, as we previously reported that triptorelin acetate and leuprolide acetate have similar effects on final oocyte maturation, we included both drugs in the study [7].

Ovarian stimulation was carried out with recombinant FSH (Gonal-F; Merck-Serono, Istanbul, Turkey) beginning on the second day of the menstrual cycle with a fixed starting dose of $225 \mathrm{IU} /$ day when the antral follicle count was $<12$ per ovary. The dose was adjusted individually according to the ovarian response. A GnRH antagonist (Cetrotide, Merck-Serono) was introduced at a dose of $0.25 \mathrm{mg} /$ day on the 6th day (a fixed antagonist protocol) and continued to be administered throughout ovarian stimulation. When at least two follicles reached $18 \mathrm{~mm}$ or three follicles reached $17 \mathrm{~mm}$ in diameter, an agent was administered to trigger final oocyte maturation. Transvaginal ultrasonography-guided oocyte retrieval was performed 
35-36 hours after final oocyte maturation. Embryo transfer was performed on the third day of oocyte retrieval. A maximum of two embryos were transferred under ultrasound guidance in accordance with national embryo transfer regulations.

All women received $90 \mathrm{mg} /$ day of vaginal micronized progesterone (Crinone $8 \%$ gel, Merck-Serono) for LPS from the day of oocyte collection until the pregnancy test performed 12 days after ET, and women with a positive pregnancy test continued this LPS regimen until 10 weeks of gestation. In addition, 1500 IU of hCG was administered on the day of oocyte retrieval for LPS in the GnRHa group.

Morphologically, top-quality embryos were those with the following characteristics: four or five blastomeres on day 2 and at least seven blastomeres on day 3 , an absence of multinucleated blastomeres, and $<20 \%$ fragmentation on days 2 and 3 after fertilization [9]. A positive pregnancy test was defined based on serum $\beta$-hCG levels measured 2 weeks after embryo transfer. Clinical pregnancy was defined as a pregnancy diagnosed via ultrasonographic visualization of one or more gestational sacs or definitive clinical signs of pregnancy. The primary outcome measures were the live birth rate, the number of good-quality embryos, and the number of mature oocytes.

\section{Statistical analysis}

Data analyses were performed using IBM SPSS ver. 21.0 (IBM Corp., Armonk, NY, USA). Samples were tested with the Shapiro-Wilk test to determine the normality of the distribution. Based on the results, parametric tests were preferred. The continuous variables were compared using one-way analysis of variance. The categorical variables were compared using the chi-square test or Fisher exact test, as appropriate. A $p$-value of $<0.05$ was considered to indicate statistical significance. When a statistically significant difference was present, a post-hoc analysis was performed between each pair of groups to identify the source of the statistical significance.

\section{Results}

The groups were comparable in baseline and demographic parameters, including age, body mass index, duration of infertility, cause of infertility, and baseline hormonal status (Table 1). All patients were normal responders from whom between 6 and 14 oocytes were collected.

The total dose of gonadotropins used, the duration of ovarian stimulation, number of oocytes collected, number of MIl oocytes, and fertilization rate were comparable among the groups (Table 2). The number of transferred embryos was $1.1 \pm 0.7$ in the GnRHa group, $1.2 \pm 0.6$ in the dual-trigger group, and $1.2 \pm 0.5$ in the $\mathrm{hCG}$ group. The total number of top-quality embryos was $3.2 \pm 2.9$ in the GnRHa group, $4.4 \pm 3.2$ in the dual-trigger group, and $2.9 \pm 2.1$ in the hCG group ( $p=0.014)$. The statistical significance stemmed from the difference between the dual-trigger and hCG groups $(p=0.011)$.

The clinical pregnancy and miscarriage rates were similar among the groups. The live birth rates per started cycle in the GnRHa, dual-trigger, and hCG trigger groups were $21.4 \%, 30.5 \%$, and $28.2 \%$, respectively $(p=0.126)$ (Table 2). No cases of OHSS were found in any of the groups.

\section{Discussion}

The present retrospective cohort study was conducted to assess the impact of the trigger of final oocyte maturation in antagonist co-treated ART cycles in normal responders. Three trigger methods were compared: a GnRHa trigger, an hCG trigger, and a dual trigger involving GnRHa and hCG. According to the results, the dual-trigger method resulted in a significantly higher number of top-quality embryos than the hCG trigger. However, the live birth rates were comparable among the groups. Although the live birth rate was lower in

Table 1. Demographic and baseline characteristics of the study groups

\begin{tabular}{|c|c|c|c|c|}
\hline Variable & $\mathrm{GnRHa}(n=56)$ & Dual trigger $(n=59)$ & hCG $(n=85)$ & $p$-value \\
\hline Age (yr) & $30.6 \pm 4.7$ & $31.4 \pm 4.2$ & $32.1 \pm 4.5$ & 0.188 \\
\hline Body mass index $\left(\mathrm{kg} / \mathrm{m}^{2}\right)$ & $23.5 \pm 4.2$ & $24.8 \pm 3.5$ & $24.9 \pm 3.7$ & 0.166 \\
\hline Basal $E_{2}(p g / m L)$ & $42.2 \pm 2.14$ & $53.4 \pm 3.8$ & $48.7 \pm 2.02$ & 0.092 \\
\hline Baseline FSH (IU/mL) & $8.2 \pm 4.4$ & $75 \pm 4.5$ & $82 \pm 3.7$ & 0.571 \\
\hline Baseline LH (IU/mL) & $4.7 \pm 2.7$ & $4.4 \pm 2.3$ & $4.4 \pm 2.1$ & 0.761 \\
\hline Duration of infertility (yr) & $6.4 \pm 3.5$ & $6.1 \pm 2.8$ & $5.8 \pm 2.4$ & 0.508 \\
\hline Cause of infertility & & & & 0.062 \\
\hline Female factor & $9(16.1)$ & $15(25.4)$ & $20(23.5)$ & \\
\hline Male factor & $31(55.4)$ & $17(28.8)$ & $38(44.7)$ & \\
\hline Unexplained & $16(16.1)$ & $27(16.1)$ & $27(16.1)$ & \\
\hline
\end{tabular}

Values are presented as mean \pm standard deviation or number (\%).

GnRHa, gonadotropin-releasing hormone agonist; $\mathrm{hCG}$, human chorionic gonadotropin; $\mathrm{E}_{2}$, estradiol; FSH, follicle-stimulating hormone; LH, luteinizing hormone. 
the GnRHa-trigger group than in the other groups, this difference was not statistically significant.

hCG has been used in routine ART cycles for final oocyte maturation. Usually, a bolus of 5,000-10,000 IU of hCG is administered to promote final oocyte maturation and ovulation. hCG primarily binds $\mathrm{LH}$ receptors to facilitate oocyte maturation. The sustained luteotrophic effect of hCG (owing to its long half-life) supports the luteal phase but also increases the risk of OHSS [1]. Although both LH and FSH surge during natural cycles to trigger ovulation, hCG lacks FSH receptor activity. Since FSH induces LH receptor formation on granulosa cells to promote oocyte maturation and cumulus expansion, an hCG trigger does not fully resemble natural oocyte maturation and ovulation [10]. Unlike hCG, a GnRHa trigger results in both LH and FSH surges [11]. Hence, the result is more similar to natural ovulation. In addition, a GnRHa trigger can also activate the $\mathrm{GnRH}$ receptors on granulosa cells, which may regulate ovulation [12]. Previously, the most important drawback of a GnRHa trigger was the associated low clinical pregnancy and high miscarriage rates $[13,14]$. Then, the Copenhagen Workshop group suggested that the luteolytic properties of GnRHa were effective in preventing OHSS, but also result in lower success rates [15]. The LH surge following a GnRHa trigger peaks more rapidly and has a shorter duration than the LH surge in a natural cycle [16]. This short surge results in oocyte maturation and ovulation but is not sufficient to support the corpus luteum [17]. This suggestion has led to the utilization of new LPS strategies, such as intensive luteal support, adjuvant low-dose hCG at the same time as GnRHa administration (dual trigger) or on the day of oocyte retrieval, and adjuvant very-low-dose hCG in the luteal phase [18-21]. Because of these strategies, the pregnancy rates have become comparable to those obtained using an hCG trigger, and OHSS rates have decreased significantly $[18,22]$.

A GnRHa trigger has become one of the preferred strategies to avoid OHSS in hyper-responder patients worldwide. However, it also confers additional benefits to ART cycles. Griffin et al. [10] reported the results of patients with a history of a $>25 \%$ proportion of immature oocytes retrieved in a prior IVF cycle who were treated with a GnRHa trigger in the subsequent cycle. The authors reported a significantly increased rate of oocyte maturation following a $\mathrm{GnRHa}$ trigger (75\%) compared to an hCG trigger (38.5\%) [10]. In another study, Kim et al. [23] randomly assigned normal responders to dual-trigger and hCG-trigger groups. They found significant improvements in the implantation rate $(24.7 \%$ vs. $14.9 \%)$, clinical pregnancy rate $(53.3 \%$ vs. $33.3 \%)$, and live birth rate (50\% vs. $30 \%)$ when a dual-trigger approach was used [23]. The suggested benefits of a dual trigger are improvements in endometrial receptivity and pregnancy rate.

In the present study, we demonstrated a significantly greater number of top-quality embryos in normal responders treated with a dual-trigger approach compared to an hCG trigger alone, but not to a GnRHa trigger alone. However, we failed to show a significant difference among the groups regarding live birth rates. While the difference was not statistically significant, the live birth rate in the $\mathrm{GnRHa}$ trigger group was remarkably lower than in the dual- and hCG-trigger groups ( $21.4 \%$ vs. $30.5 \%$ vs. $28.2 \%$, respectively). The mean numbers of oocytes retrieved and MII oocytes were comparable among the groups in our study. However, the total number of Mll oocytes tended to be greater in the dual-trigger group than in the other groups. Hence, the results of this study should be interpreted with

Table 2. Comparison of cycle characteristics and outcome parameters between the study groups

\begin{tabular}{|c|c|c|c|c|}
\hline Variable & $\mathrm{GnRHa}(\mathrm{n}=56)$ & Dual trigger $(n=59)$ & hCG $(n=85)$ & $p$-value \\
\hline Duration of stimulation (day) & $9.4 \pm 1.3$ & $9.5 \pm 2.4$ & $9.3 \pm 1.7$ & 0.913 \\
\hline Total dose of gonadotropins (IU) & $2,442 \pm 975$ & $2,390 \pm 1,049$ & $2,725 \pm 932$ & 0.086 \\
\hline Oestradiol levels on the day of trigger $(\mathrm{pg} / \mathrm{mL})$ & $1,640 \pm 1,205$ & $1,916 \pm 1,128$ & $1,406 \pm 928$ & $0.026^{\mathrm{a})}$ \\
\hline Number of retrieved oocytes & $7.4 \pm 4.9$ & $9.2 \pm 5.3$ & $7.6 \pm 4.5$ & 0.087 \\
\hline Number of MIl oocytes & $6.2 \pm 4.2$ & $7.2 \pm 4.7$ & $5.6 \pm 3.7$ & 0.095 \\
\hline Fertilization rate (\%) & $69 \pm 42$ & $70 \pm 33$ & $62 \pm 29$ & 0.500 \\
\hline Number of top-quality embryos & $3.2 \pm 2.9$ & $4.4 \pm 3.2$ & $2.9 \pm 2.1$ & $0.014^{\mathrm{b})}$ \\
\hline Number of transferred embryos & $1.1 \pm 0.7$ & $1.2 \pm 0.6$ & $1.2 \pm 0.5$ & 0.291 \\
\hline Endometrial thickness on the day of embryo transfer $(\mathrm{mm})$ & $10.4 \pm 1.7$ & $10.4 \pm 1.8$ & $10.7 \pm 1.6$ & 0.490 \\
\hline Positive pregnancy test & $15(26.8)$ & $20(33.9)$ & $31(36.5)$ & 0.141 \\
\hline Clinical pregnancy & $13(23.2)$ & $20(33.9)$ & $26(30.6)$ & 0.112 \\
\hline Miscarriage & $1(1.8)$ & $2(3.4)$ & $2(2.4)$ & 0.148 \\
\hline Live birth & $12(21.4)$ & $18(30.5)$ & $24(28.2)$ & 0.126 \\
\hline
\end{tabular}

Values are presented as mean \pm standard deviation or number (\%).

$\mathrm{GnRHa}$, gonadotropin-releasing hormone agonist; $\mathrm{hCG}$, human chorionic gonadotropin; MII, metaphase II.

Significant difference between the dual-trigger and hCG groups: ${ }^{\mathrm{a}} p<0.001,{ }^{\mathrm{b}} p=0.011$. 
caution, as the number of top-quality embryos could be affected by the number of MII oocytes. Additionally, the greater number of MII oocytes could result from the type of trigger, as well the higher peak oestradiol levels in the dual-trigger group.

Recently, in a retrospective cohort study, Zhou et al. [24] demonstrated that a dual trigger results in greater numbers of two-pronuclear embryos, embryos available, and high-quality embryos. They also reported nonsignificant trends towards higher implantation, clinical pregnancy, and live delivery rates in the dual-trigger group compared to the hCG-trigger group [24]. In another recent study, Beck-Fruchter et al. [8] compared the results following a Gn$\mathrm{RHa}$ trigger and an hCG trigger in normal responders. The authors reported similar outcomes regarding the number of oocytes, oocyte maturation rate, implantation rate, and live birth rate [8]. Our results aligned with the results of these two recent studies in that a dual trigger was superior to an hCG trigger alone. However, we showed no significant advantage of a dual trigger over a $\mathrm{GnRHa}$ trigger alone in normal responders with regard to the number of top-quality embryos.

The most important strength of our study was its comparison of three different types of ovulation triggers. To the best of our knowledge, this is the first study that included a comparison between a dual trigger and a GnRHa trigger in normal responders. The systematic exploration of individual parameters may also add credence to our observations. The main limitations of the present study were its retrospective nature and small sample size. For instance, the non-significant differences among the dual-trigger group and the other groups regarding oocyte numbers, MII numbers, and (in particular) live birth rates may be reflections of the small sample size. Considering the relatively low live birth rate found in the GnRHa-trigger group, one could assume that a significant difference might be present if a larger cohort were used. However, when interpreting the results, the smaller number of patients and higher peak oestradiol levels in the dual-trigger group should be noted as limitations of this study. Also, the non-randomized case selection makes our study unlikely to be involved in future meta-analyses. Another limitation of our study was the absence of frozen-thawed cycles in the analyses. It is plausible that the inclusion of such cycles would result in better outcomes. Finally, the lack of a sample size calculation should also be noted as a limitation of this study.

In conclusion, in terms of the number of top-quality embryos in normal responders, a dual-trigger approach seems superior to an hCG trigger alone, but not superior to a GnRHa trigger alone. However, no clinical benefit seems to exist in terms of live birth rates. Future randomized controlled trials in large cohorts and meta-analyses are needed to clarify the exact impact of the trigger of final oocyte maturation in normal responders undergoing ART cycles.

\section{Conflict of interest}

No potential conflict of interest relevant to this article was reported.

\section{ORCID}

Yavuz Emre Şükür

Hasan Ulubaşoğlu

Fatma Ceylan illhan

Bülent Berker

Murat Sönmezer

Cem Somer Atabekoğlu

Ruşen Aytaç

Batuhan Özmen

https://orcid.org/0000-0003-0815-3522

https://orcid.org/0000-0001-9157-0612

https://orcid.org/0000-0003-1919-8072

https://orcid.org/0000-0001-7346-7128

https://orcid.org/0000-0001-6101-1414

https://orcid.org/0000-0003-0264-0709

https://orcid.org/0000-0002-2644-545X

https://orcid.org/0000-0002-4504-669X

\section{Author contributions}

Conceptualization: BÖ. Data curation: HU, FCI. Formal analysis: YEŞ. Methodology: YEŞ. Visualization: BB, MS, CSA, RA. Writing-original draft: YEŞ. Writing-review \& editing: BÖ.

\section{References}

1. Cerrillo M, Pacheco A, Rodríguez S, Gómez R, Delgado F, Pellicer A, et al. Effect of GnRH agonist and hCG treatment on VEGF, angiopoietin-2, and VE-cadherin: trying to explain the link to ovarian hyperstimulation syndrome. Fertil Steril 2011;95:2517-9.

2. Youssef MA, Van der Veen F, Al-Inany HG, Mochtar MH, Griesinger G, Nagi Mohesen M, et al. Gonadotropin-releasing hormone agonist versus HCG for oocyte triggering in antagonist-assisted reproductive technology. Cochrane Database Syst Rev 2014;(10): CD008046.

3. Gonen Y, Balakier H, Powell W, Casper RF. Use of gonadotropin-releasing hormone agonist to trigger follicular maturation for in vitro fertilization. J Clin Endocrinol Metab 1990;71:918-22.

4. Shalev E, Geslevich Y, Ben-Ami M. Induction of pre-ovulatory luteinizing hormone surge by gonadotrophin-releasing hormone agonist for women at risk for developing the ovarian hyperstimulation syndrome. Hum Reprod 1994;9:417-9.

5. Kol S, Humaidan P. GnRH agonist triggering: recent developments. Reprod Biomed Online 2013;26:226-30.

6. Benadiva C, Engmann L. Luteal phase support after gonadotropin-releasing hormone agonist triggering: does it still matter? Fertil Steril 2018;109:763-7.

7. Sukur YE, Ozmen B, Ozdemir ED, Seval MM, Kalafat E, Sonmezer M, et al. Final oocyte maturation with two different GnRH agonists in 
antagonist co-treated cycles at risk of ovarian hyperstimulation syndrome. Reprod Biomed Online 2017;34:5-10.

8. Beck-Fruchter R, Baram S, Geslevich Y, Weiss A. Gonadotropin releasing hormone agonist final oocyte maturation and human chorionic gonadotropin as exclusive luteal support in normal responders. Gynecol Obstet Invest 2019;84:27-34.

9. Van Royen E, Mangelschots K, De Neubourg D, Valkenburg M, Van de Meerssche M, Ryckaert G, et al. Characterization of a top quality embryo, a step towards single-embryo transfer. Hum Reprod 1999;14:2345-9.

10. Griffin D, Feinn R, Engmann L, Nulsen J, Budinetz T, Benadiva C. Dual trigger with gonadotropin-releasing hormone agonist and standard dose human chorionic gonadotropin to improve oocyte maturity rates. Fertil Steril 2014;102:405-9.

11. Engmann L, Benadiva C, Humaidan P. GnRH agonist trigger for the induction of oocyte maturation in GnRH antagonist IVF cycles: a SWOT analysis. Reprod Biomed Online 2016;32:274-85.

12. Maggi R, Cariboni AM, Marelli MM, Moretti RM, Andre V, Marzagalli $\mathrm{M}$, et al. $\mathrm{GnRH}$ and $\mathrm{GnRH}$ receptors in the pathophysiology of the human female reproductive system. Hum Reprod Update 2016;22:358-81.

13. Humaidan $P$, Bredkjaer HE, Bungum L, Bungum M, Grondahl ML, Westergaard L, et al. GnRH agonist (buserelin) or hCG for ovulation induction in $\mathrm{GnRH}$ antagonist IVF/ICSI cycles: a prospective randomized study. Hum Reprod 2005;20:1213-20.

14. Kolibianakis EM, Schultze-Mosgau A, Schroer A, van Steirteghem A, Devroey P, Diedrich K, et al. A lower ongoing pregnancy rate can be expected when $\mathrm{GnRH}$ agonist is used for triggering final oocyte maturation instead of HCG in patients undergoing IVF with GnRH antagonists. Hum Reprod 2005;20:2887-92.

15. Humaidan P, Kol S, Papanikolaou EG; Copenhagen GnRH Agonist Triggering Workshop Group. GnRH agonist for triggering of final oocyte maturation: time for a change of practice? Hum Reprod Update 2011;17:510-24.

16. Itskovitz J, Boldes R, Levron J, Erlik Y, Kahana L, Brandes JM. Induction of preovulatory luteinizing hormone surge and prevention of ovarian hyperstimulation syndrome by gonadotropin-releasing hormone agonist. Fertil Steril 1991;56:213-20.

17. Zelinski-Wooten MB, Lanzendorf SE, Wolf DP, Chandrasekher YA,
Stouffer RL. Titrating luteinizing hormone surge requirements for ovulatory changes in primate follicles. I. Oocyte maturation and corpus luteum function. J Clin Endocrinol Metab 1991;73:577-83.

18. Humaidan P, Ejdrup Bredkjaer H, Westergaard LG, Yding Andersen C. 1,500 IU human chorionic gonadotropin administered at oocyte retrieval rescues the luteal phase when gonadotropin-releasing hormone agonist is used for ovulation induction: a prospective, randomized, controlled study. Fertil Steril 2010;93:84754.

19. Shapiro BS, Daneshmand ST, Garner FC, Aguirre M, Thomas S. Gonadotropin-releasing hormone agonist combined with a reduced dose of human chorionic gonadotropin for final oocyte maturation in fresh autologous cycles of in vitro fertilization. Fertil Steril 2008;90:231-3.

20. Haas J, Kedem A, Machtinger R, Dar S, Hourvitz A, Yerushalmi G, et al. HCG (1500IU) administration on day 3 after oocytes retrieval, following $\mathrm{GnRH}$-agonist trigger for final follicular maturation, results in high sufficient mid luteal progesterone levels: a proof of concept. J Ovarian Res 2014;7:35.

21. Andersen CY, Elbaek HO, Alsbjerg B, Laursen RJ, Povlsen BB, Thomsen L, et al. Daily low-dose hCG stimulation during the luteal phase combined with GnRHa triggered IVF cycles without exogenous progesterone: a proof of concept trial. Hum Reprod 2015; 30:2387-95.

22. Humaidan P, Polyzos NP, Alsbjerg B, Erb K, Mikkelsen AL, Elbaek $\mathrm{HO}$, et al. GnRHa trigger and individualized luteal phase hCG support according to ovarian response to stimulation: two prospective randomized controlled multi-centre studies in IVF patients. Hum Reprod 2013;28:2511-21.

23. Kim CH, Ahn JW, You RM, Kim SH, Chae HD, Kang BM. Combined administration of gonadotropin-releasing hormone agonist with human chorionic gonadotropin for final oocyte maturation in $\mathrm{GnRH}$ antagonist cycles for in vitro fertilization. J Reprod Med 2014;59:63-8.

24. Zhou X, Guo P, Chen X, Ye D, Liu Y, Chen S. Comparison of dual trigger with combination GnRH agonist and hCG versus hCG alone trigger of oocyte maturation for normal ovarian responders. Int J Gynaecol Obstet 2018;141:327-31. 